\title{
Particle filtering for indoor RFID tag tracking
}

\author{
Vladimir Savic, Akshay Athalye, Miodrag Bolic and Petar M. Djuric
}

\section{Linköping University Post Print}

N.B.: When citing this work, cite the original article.

C2011 IEEE. Personal use of this material is permitted. However, permission to reprint/republish this material for advertising or promotional purposes or for creating new collective works for resale or redistribution to servers or lists, or to reuse any copyrighted component of this work in other works must be obtained from the IEEE.

Vladimir Savic, Akshay Athalye, Miodrag Bolic and Petar M. Djuric, Particle filtering for indoor RFID tag tracking, 2011, Proc. of IEEE Statistical Signal Processing Workshop (SSP), 193-196.

http://dx.doi.org/10.1109/SSP.2011.5967656

Postprint available at: Linköping University Electronic Press http://urn.kb.se/resolve?urn=urn:nbn:se:liu:diva-81324 


\title{
PARTICLE FILTERING FOR INDOOR RFID TAG TRACKING
}

\author{
Vladimir Savic, ${ }^{\dagger}$ Akshay Athalye, ${ }^{*}$ Miodrag Bolic,${ }^{\ddagger}$ and Petar M. Djuric ${ }^{* *}$
}

\author{
† Signal Processing Applications Group, Universidad Politecnica de Madrid, Spain, e-mail: vladimir@gaps.ssr.upm.es \\ * Center of Excellence in Wireless and Information Technology, Stony Brook University, USA, e-mail: akshay.athalye@ cewit.stonybrook.edu \\ ¥ School of Information Technology and Engineering, University of Ottawa, Canada, e-mail: mbolic@ site.uottawa.ca \\ ** Department of Electrical \& Computer Engineering, Stony Brook University, USA, e-mail:djuric@ece.sunysb.edu
}

\begin{abstract}
In this paper, we propose a particle filtering (PF) method for indoor tracking using radio frequency identification (RFID) based on aggregated binary measurements. We use an Ultra High Frequency (UHF) RFID system that is composed of a standard RFID reader, a large set of standard passive tags whose locations are known, and a newly designed, special semi-passive tag attached to an object that is tracked. This semi-passive tag has the dual ability to sense the backscatter communication between the reader and other passive tags which are in its proximity and to communicate this sensed information to the reader using backscatter modulation. We refer to this tag as a sense$a$-tag (ST). Thus, the ST can provide the reader with information that can be used to determine the kinematic parameters of the object on which the ST is attached. We demonstrate the performance of the method with data obtained in a laboratory environment.
\end{abstract}

Index Terms - particle filtering, tracking, RFID, tags

\section{INTRODUCTION}

Radio Frequency IDentification (RFID) is a well-known technology for real-time identification of objects and people. Besides identification, RFID can also be used for localization and tracking, which can enable a number of applications involving tracking of people, robots and other mobile objects such as forklifts and carts used in warehouses [1]. Methods for RFID localization and tracking can be broadly classified into three categories [2]: i) distance-based, ii) scene-analysis, and iii) proximity-based. Distance-based methods rely on range measurements that can be Received Signal Strength (RSS), Time Of Arrival (TOA), or Time Difference of Arrival (TDOA). Scene-analysis methods consist of two phases. First, environmental information (fingerprints) is acquired. Then, the target location is estimated by matching the measurements with the stored fingerprints. All of these methods require some kind of calibration, and therefore they are not suitable for environments with dynamic changes. Proximity-based methods employ a different approach, using binary information related to the presence or absence of the target within the ranges of reference tags.

This work was supported by the European Commission under the grants FP7-ICT-2009-4-248894-WHERE-2 and FP7-ICT-223994-N4C, the NSF SBIR grant IIP-0912774, the Spanish Ministry of Science and Innovation under grants TEC2009-14219-C03-01, TEC2010-21217-C02-02-CR4HFDVL, TEC 2008-04644-E, and program CONSOLIDER-INGENIO 2010 under grant CSD2008-00010 COMONSENS. We also thank the support of the Center of Excellence of Wireless and Information Technology. V. S. was supported by the FPU fellowship from the Spanish Ministry of Science and Innovation. P. M. D. was supported by NSF under Award CCF-1018323 and by the ONR under Award N00014-09-1-1154.
In this paper, we propose a novel particle filtering method (called PF-BIN), based on aggregated binary measurements for indoor RFID tracking. ' We use a passive RFID system, which is composed of a standard UHF, ISO 18000-6C (Class1, Gen2) compliant RFID reader, a large set of standard passive tags whose locations are known, and a specially designed semi-passive tag, which is attached to an object that is tracked. The new tag, referred to as sense-a-tag (ST) [4], can sense backscatter communication between the reader and passive tags that are in its proximity and can communicate the sensed information to the reader using backscatter modulation. The ST will detect closer tags more often than distant ones in a fixed number of reader queries, and this information can be used for tracking. Therefore, our method for tracking belongs to the class of proximity-based methods.

The paper is organized as follows. In Section 2, we briefly describe the ST and the system used for tracking. In Section 3, we present the methods for indoor tag tracking. The experimental results are shown in Section 4. We conclude the paper with some final remarks in Section 5.

\section{HARDWARE DESCRIPTION}

Our approach is based on the use of STs in conjunction with a standard reader-tag RFID system. Passive tags with known locations are deployed in the area of tracking. The ST is placed on the object that needs to be tracked, and an RFID reader with one or more antennas is positioned so as to "illuminate" the tracking area. The ST has the following capabilities: i) to detect and decode backscatter signals from RFID tags in its proximity and ii) to communicate with the reader using backscatter modulation. For more on the characteristics of the ST, see $[3,4]$.

Passive and semi-passive RFID tags communicate with a reader via a weak reflected backscatter. This backscatter is further affected by multipath reflections and other ambient interferences in cluttered indoor environments like warehouses, retail stores, libraries, and offices [5]. This results in a low signal-to-noise ratio (SNR) of the tag's response received by the reader. Consequently, conventional distance-based techniques for localization become highly inaccurate and unreliable. Our ST based approach leads to a more robust solution, since it relies on capturing the weak backscatter signal in the close proximity of the tags, where it is stronger rather than at a distance where it is weaker and affected more by the above interferences.

The ST communicates passively without an on board radio. However, an on board battery is used for powering up the ST circuitry, thus making it a semi-passive device. The ST incorporates

\footnotetext{
${ }^{1}$ For indoor localization using an ST, see [3]
} 
a novel locator protocol, which is fully compatible with the EPC (Electronic Product Code) Global Class 1 Gen 2 standard. This protocol enables the ST to communicate with a standard reader and conveys binary information about the presence or absence of a responding tag in its proximity. The protocol specifies two states of operation for the ST. In the first state or the listen state, the ST listens for backscattering tags in its vicinity. In the second state or the respond state, the ST itself functions as an RFID tag and conveys the information of the tags detected when it was in the listen state as part of its EPC ID payload. The transition between the two states is done using the Select function provided by the 18000-6C compliant RFID reader. The tracking algorithm (see Section 3), which runs on the host computer that also controls the reader, uses aggregated binary information from successive query rounds.

\section{INDOOR TRACKING USING RFID SYSTEM}

Let us assume that we have $K$ reference (passive) tags with known two-dimensional (2D) positions, $l_{k}(k=1,2, \cdots, K)$ and one ST attached to an object with an unknown position and velocity $x_{t}$ at time $t$. A reference tag can be detected by an ST with probability $p_{k, t}$. This probability depends on various factors, but primarily on the distance between the reference tag and the ST, orientation, and the power of the reader [5]. This probability is easily estimated by counting the number of detections of a tag by an ST in a fixed number of reader queries. Using this observation, our goal is to estimate $x_{t}$ at each time $t$.

\subsection{Standard Bayesian solutions}

We use the following discrete state-space model:

$$
\begin{gathered}
x_{t+1}=A x_{t}+B u_{t} \\
y_{t}=C x_{t}+v_{t}
\end{gathered}
$$

where $x_{t}=\left[\begin{array}{llll}x_{1, t} & x_{2, t} & \dot{x}_{1, t} & \dot{x}_{2, t}\end{array}\right]^{T}$ is the state vector at time $t$, which includes the position and velocity of the ST that we want to estimate, $u_{t}=\left[\begin{array}{ll}u_{1, t} & u_{2, t}\end{array}\right]^{T}$ is the process noise (which accounts for the variation of the speed), $y_{t}=\left[\begin{array}{ll}y_{1, t} & y_{2, t}\end{array}\right]^{T}$ is observation at time $t$, and $v_{t}=\left[v_{1, t} v_{2, t}\right]^{T}$ is observation noise. The observation is given as $y_{t}=\sum_{k} p_{k, t} l_{k}$, i.e., the weighted average of the positions of the detected tags. We have already shown in [3] that this position estimate is more accurate than other estimates found either by non-weighted average, or simply by association with the nearest reference point. It is also worth noting that, since our observations represent static position estimates, our model is linear (in contrast to distance-based methods). Given this observation, the sampling period $T_{S}$, and assuming random motion of the target, we can define the matrices $A$, $B$, and $C$ as follows:

$$
\begin{aligned}
A & =\left[\begin{array}{cccc}
1 & 0 & T_{S} & 0 \\
0 & 1 & 0 & T_{S} \\
0 & 0 & 1 & 0 \\
0 & 0 & 0 & 1
\end{array}\right], B=\left[\begin{array}{ll}
T_{S} & 0 \\
0 & T_{S} \\
1 & 0 \\
0 & 1
\end{array}\right] . \\
C & =\left[\begin{array}{llll}
1 & 0 & 0 & 0 \\
0 & 1 & 0 & 0
\end{array}\right]
\end{aligned}
$$

We apply the Bayesian approach to solve this tracking problem and in particular, we use Kalman filtering and particle filtering. At time $t$, our goal is to estimate the posterior distribution $p\left(x_{t} \mid y_{1: t}\right)$ given the prior $p\left(x_{t-1} \mid y_{1: t-1}\right)$ (initially, $p\left(x_{0} \mid y_{0}\right)=p\left(x_{0}\right)$ is available), the state evolution $p\left(x_{t} \mid x_{t-1}\right)$ (defined by the motion model

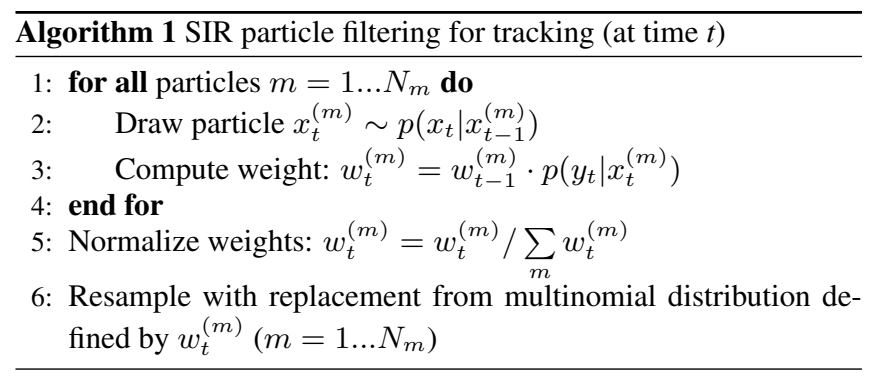

(1)), and the likelihood function $p\left(y_{t} \mid x_{t}\right)$ (defined by the measurement model (2)). This posterior can be found by following the prediction and filtering equations [6]:

$$
\begin{gathered}
p\left(x_{t} \mid y_{1: t-1}\right)=\int p\left(x_{t} \mid x_{t-1}\right) p\left(x_{t-1} \mid y_{1: t-1}\right) d x_{t-1} \\
p\left(x_{t} \mid y_{1: t}\right) \propto p\left(y_{t} \mid x_{t}\right) p\left(x_{t} \mid y_{1: t-1}\right) .
\end{gathered}
$$

A standard closed-form solution can be found using traditional Kalman filtering (KF) [7], assuming that the model is linear (as in our case), and that $u_{t}$ and $v_{t}$ are drawn from Gaussian distributions. The estimation of the process noise $u_{t}$ is generally very difficult (it requires an accelerometer or a similar device attached to the target). Thus, we approximate $u_{t}$ by a Gaussian distribution. To make the process reliable, we need to find an upper bound of the true noise, e.g., by injecting enough uncertainty into the covariance matrix. However, the measurement noise $v_{t}$ can be easily obtained using real samples. Generally, we cannot expect (especially, in indoor environment) that this noise $v_{t}$ is Gaussian, so KF is not an optimal solution for our problem.

Therefore, we apply the particle filtering (PF) method [6] in which we represent the posterior distribution by a set of random samples (particles) with associated weights. We apply the wellknown sample-importance-resampling (SIR) method (called PFSIR). In this method, the particles are drawn from $p\left(x_{t} \mid x_{t-1}\right)$, then weighted by the likelihood function, $p\left(y_{t} \mid x_{t}\right)$, and finally, resampled in order to avoid the degeneracy problem (the situation in which all but one particle have negligible weights). The PF-SIR method is summarized in Alg. 1.

Note that the likelihood function does not have a parametric form, and therefore we want to find its kernel density estimate (KDE) [8]. Namely, given a set of $N_{i}$ calibration samples $v_{t}^{i}=y_{t}^{i}-C x_{t}^{i}$, we have:

$$
p\left(v_{t}\right)=\sum_{i} \mathcal{K}_{h}\left(v_{t}-v_{t}^{i}\right)
$$

where $\mathcal{K}_{h}$ is the commonly used spherically symmetric Gaussian kernel: $\mathcal{K}_{h}(x)=\mathcal{N}(x, 0, h I)$, and $h$ is the bandwidth which controls the variance. To find $h$, we use the generalized cross entropy (GCE) estimator [9], which provides very accurate estimates. This kernel can be found offline prior to tracking. However, if the RFID system is fast enough to provide $N_{i}$ samples during the sampling period $\left(T_{S}\right)$ and also to compute (5), the likelihood function can be obtained online, at each time frame. We can see that the main drawback of this method is high complexity.

\subsection{Improved PF method (PF-BIN)}

We propose a model for the number of detections of a tag by an ST and show how we proceed with particle filtering. First, we model the 
probability of detection of a tag by an ST according to

$$
p=\frac{1}{1+e^{\alpha\left(d-d_{0}\right)}}
$$

where $d$ is the distance between a tag and the ST, and $\alpha>0, d_{0}>0$ are parameters of the model, with $d_{0}$ being the distance at which the probability of detection is equal to $1 / 2$, and $\alpha$ being the parameter which determines the steepness of the function.

Our measurements represent the number of times a tag is detected by an ST in $N$ query rounds. We assume that during the $N$ query rounds, the location of the object with the ST has not changed much (recall that the object with the attached ST is moving). Let the number of detections of the $k$ th tag be equal to $n_{k}$. Then the probability of $n_{k}$ is modeled by the binomial distribution, i.e.,

$$
P\left(n_{k}\right)=\left(\begin{array}{c}
N \\
n_{k}
\end{array}\right) p_{k}^{n_{k}}\left(1-p_{k}\right)^{N-n_{k}}
$$

where $p_{k}$ is given by (6) with $d$ replaced by $d_{k}$, the distance between the ST and the $k$ th tag. In the field, there are total of $K$ tags and for each of them we have a number of detections $n_{k} \in\{0,1, \cdots, N\}$, $k=1,2, \cdots, K$.

Under the assumption that the parameters of the model in (6) are known (they are estimated offline), we proceed with particle filtering as follows (note that we also assume that at time $t-1$ we have the set of particles $\left.x_{t-1}^{(m)}\right)$ :

Step 1: Propagate the particles by using the prior, that is,

$$
x_{t}^{(m)} \sim p\left(x_{t} \mid x_{t-1}^{(m)}\right) .
$$

Step 2: Compute the likelihood of the particles $x_{t}^{(m)}$ given the measurements $y_{t}=\left[\begin{array}{llll}n_{1, t} & n_{2, t} & \cdots & n_{K, t}\end{array}\right]^{\top}$. The likelihood function is given by

$$
\begin{aligned}
p\left(y_{t} \mid x_{t}^{(m)}\right) & \\
& \left.=\prod_{k=1}^{K}\left(\begin{array}{c}
N \\
n_{k}
\end{array}\right) p_{k, t}^{(m)}\right)^{n_{k, t}}\left(1-p_{k, t}^{(m)}\right)^{\left(N-n_{k, t}\right)}
\end{aligned}
$$

where

$$
p_{k, t}^{(m)}=\frac{1}{1+e^{\alpha\left(d_{k, t}^{(m)}-d_{0}\right)}}
$$

and $d_{k, t}^{(m)}$ is the distance between the ST (whose location is defined by the particle $x_{t}^{(m)}$ ) and the $k$ th tag at time $t$. We note that the weights of the particles are

$$
w_{t}^{(m)} \propto p\left(y_{t} \mid x_{t}^{(m)}\right) .
$$

Step 3: Resample with replacement.

\section{EXPERIMENTAL RESULTS}

Figure 1 shows our experimental setup. We deployed 9 reference tags in an area of $3 \mathrm{~m} \times 1.6 \mathrm{~m}$. The reader antenna was at a distance of about $2 \mathrm{~m}$ from the center of the area, and its power level was set to $28 \mathrm{dBm}$. The ST was placed on a chair with wheels that could be moved easily. Our objective was to track the ST during a period of $6 \mathrm{~s}\left(T_{s} \approx 0.7 s\right)$. In the experiment the speed of the movement was approximately constant.

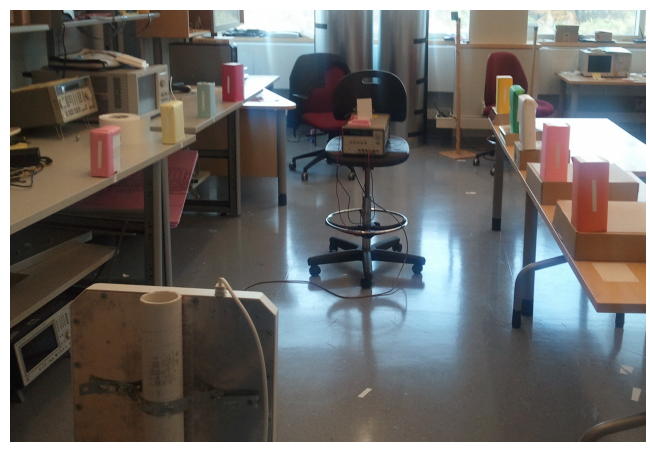

Fig. 1: Experimental setup. There were 9 reference points (shown by the standing boxes), one reader (shown antenna in foreground), and one ST (on the chair) which represents the target.

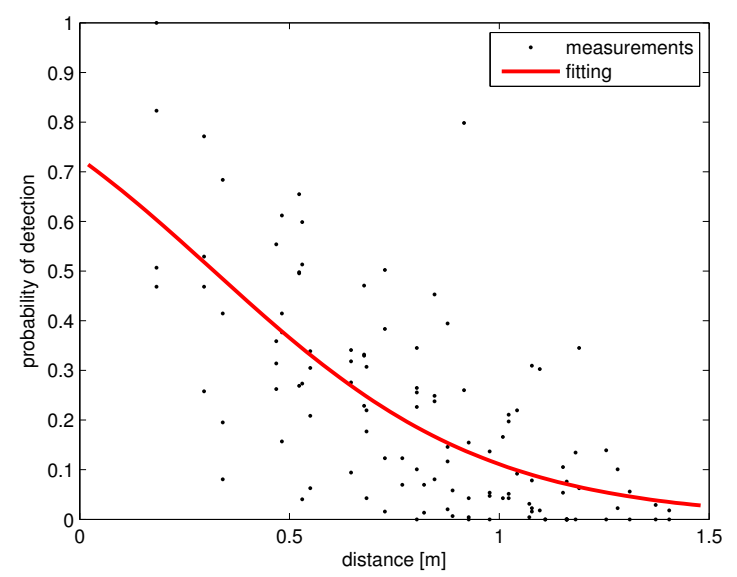

Fig. 3: Samples of probability of detection and the corresponding fitting.

In the first set of experiments, our goal was to obtain calibration samples $^{2}$ used for estimation of the likelihood (for PF-SIR), measurement covariance matrix (for KF) and probability of detection (for PF-BIN). To that end, we acquired 20 independent measurements at 20 grid points. Using these samples, we obtained empirical KDE of the measurement noise used for the PF-SIR method. For the KF method, we estimated the measurement covariance matrix $R=\operatorname{diag}(0.025,0.027)$, and assumed (without measuring) that the process covariance matrix was given by $Q=\operatorname{diag}(0.2 \mathrm{~V}, 0.2 \mathrm{~V})$ where $V$ is the speed of movement. Finally, for the PF-BIN method, we estimated the parameters of our model for probability of detection as $\widehat{\alpha}=3.059$ and $\widehat{d}_{0}=0.32 \mathrm{~m}$. The estimation was performed based on fitting with the exponential curve as shown in Figure 3. Having defined all the parameters, we tracked the ST over a number of different tracks. We applied the KF, PF-SIR and PF-BIN methods. The results for two tracks are shown in Figure 2.

Finally, we conducted simulations of 100 random tracks. We used the same model, obtained from the real data. We changed the number of reference tags $(K=16)$, the sampling period $\left(T_{s}=\right.$ $0.3)$, and the deployment area $(4 \mathrm{~m} \times 4 \mathrm{~m})$. According to Figure 4, where we show the averaged RMSE over the 100 tracks, the PFBIN consistently performed better than the PF-SIR. On the other hand, the KF had the worst performance during some initial period,

\footnotetext{
${ }^{2}$ Due to the complex location protocol (see Section 2), our RFID system was not fast enough to obtain the likelihood online.
} 


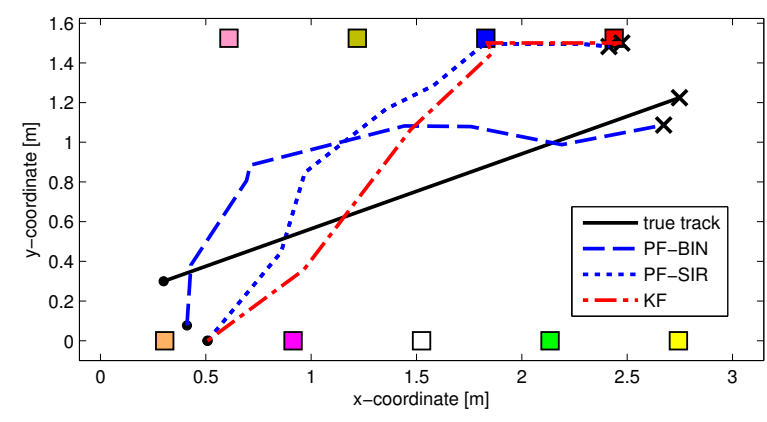

(a)

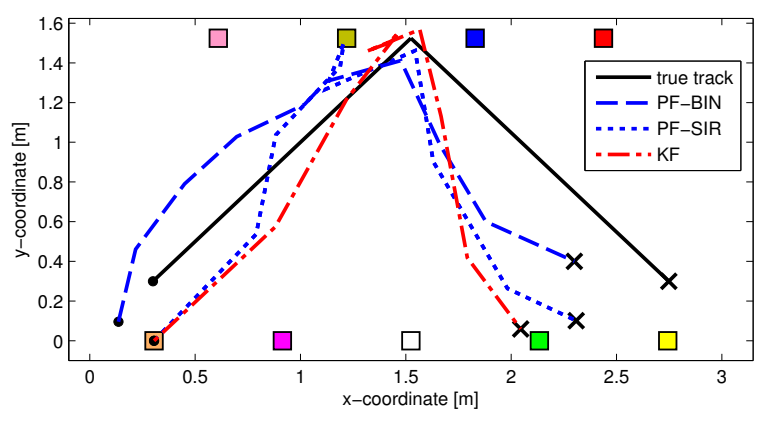

(b)

Fig. 2: Illustration of results of tracking for two different tracks. The starting point of the target is marked with a dot, and the destination point with an $\mathrm{x}$.

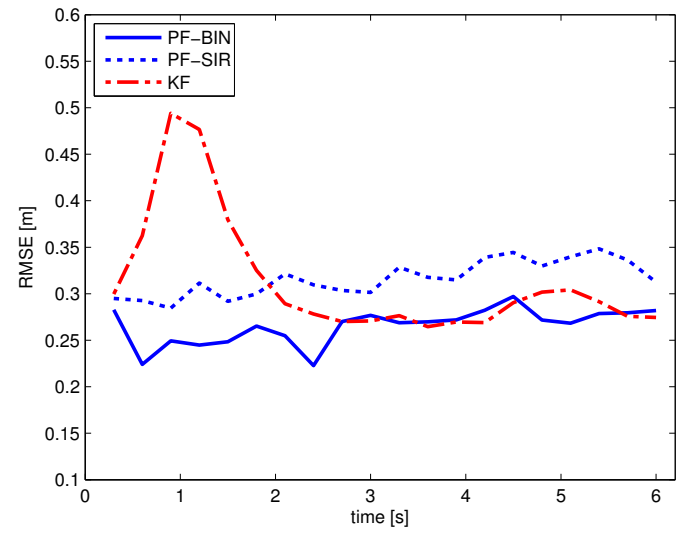

Fig. 4: Comparison of the RMSEs of the three methods.

probably because of the setup time that is necessary for parameter tuning. In Figure 5, we plotted the cumulative distribution functions (CDF) of the errors of each of the methods. As we can see , PF-BIN performed the best of all methods.

Regarding complexity, we found that PF-BIN was twice faster than the PF-SIR, but about 10 times slower than the KF. Thus, one may conclude that the KF is an option for a low-cost application where high accuracy is not crucial. However, if one wants to have a robust algorithm, PF-based methods should be applied. In our experiments, we did not detect large outliers, but in general, they can be expected.

\section{CONCLUSIONS}

In this paper, we presented three methods for RFID based tracking of objects using aggregated binary measurements in indoor environments. They include two PF methods and a Kalman filtering method. The two PF methods are based on two different observation models. With simulations and experimentation with real data obtained using a novel semi-passive RFID system, we showed that the PF method referred to as PF-BIN performed the best in terms of both RMSE and CDF. There remain a few issues we plan to explore. They include the implementation of a real-time PF-BIN method that estimates the parameters $\alpha$ and $d_{0}$ online.

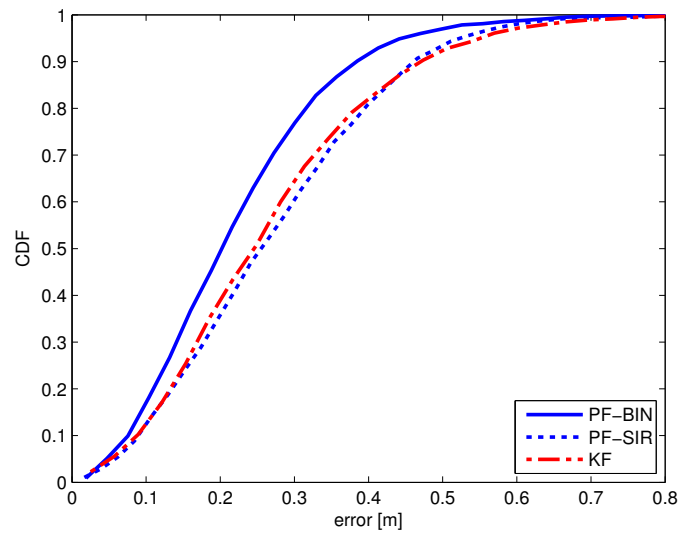

Fig. 5: Comparison of the CDFs of position errors of the three methods.

\section{REFERENCES}

[1] T. Sanpechuda, L. Kovavisaruch, "A review of RFID localization: Applications and techniques," in IEEE Proc. of International Conference on ECTI-CON, pp.769-772, May 2008.

[2] M. Bouet, and A.L. dos Santos, "RFID tags: Positioning principles and localization techniques," in IEEE Proc. of IFIP Wireless Days, pp. 1-5, Nov. 2008

[3] A. Athalye, V. Savić, M. Bolić, P. M. Djurić, "A radio frequency identification system for accurate indoor localization," to appear in IEEE Proc. of ICASSP 2011, May 2011.

[4] P. M. Djurić and A. Athalye, "RFID system and method for localizing and tracking a moving object with an RFID tag," Patent, Approved on: 2010-06-24; Application Number: 11799257, 2007.

[5] M. Bolic, A. Athalye, T. Li, "Performance of passive UHF RFID systems in practice," in M. Bolic, D. Simplot-Ryl, I. Stojmenovic, RFID Systems: Research trends and challenges, edited book, Wiley 2010.

[6] M. S. Arulampalam, S. Maskell, N. Gordon, and T. Clapp, "A tutorial on particle filters for online nonlinear/non-Gaussian Bayesian tracking," IEEE Transactions on Signal Processing, vol. 50, issue 2, pp. 174-188, February 2002.

[7] G. Welch and G. Bishop, "An introduction to the Kalman Filter," Technical Report 95-041, 2006.

[8] B.W. Silverman, Density Estimation for Statistics and Data Analysis, Chapman and Hall, New York, 1986.

[9] Z.I. Botev, "A novel nonparametric density estimator," Technical Report, The University of Queensland, Australia, 2006. 